\title{
A Ubiquitous System for Smart Reasoning for Well-Being at Home and at Work
}

\author{
Steven Bosems \\ Faculty of EEMCS \\ University of Twente \\ Enschede, The Netherlands \\ Email: s.bosems@utwente.nl
}

\begin{abstract}
The lifestyle of the Dutch workforce is degrading. Unhealthy habits cause both physical and psychological problems, putting a strain on the individual's well-being. In order to conquer both of these, a system will be created that will coach its user to improve their lifestyle through a better diet and promoting physical activity in order to improve their feeling of well-being. However, requirements engineering is troublesome in this domain. We propose ways to conquer these requirements engineering problems using model-driven engineering techniques.
\end{abstract}

Index Terms-Health information management, Behavioral change, Requirements engineering, Model Driven Engineering

\section{INTRODUCTION}

In a yearly report [1], the Dutch Labour Inspectorate publishes the current state of the Dutch workforce. Among others, the report includes among others statistical information about the Dutch employees, working conditions, time and place independent working, durable ways to use human resources and the health of the employees. Data from this last theme causes concern.

From the report we can derive that Dutch employees have an unhealthy lifestyle: $50 \%$ exercise too little, $44 \%$ cope with overweight, $28 \%$ smoke, of which over $82 \%$ smoke more than 10 cigarets or cigars per day, $17 \%$ do not have breakfast, and $5 \%$ abuse alcohol.

As we learn from this report, an unhealthy lifestyle has consequences on the way people perceive their health, but also on sick leave and even how they interact with others. As a result, bad habits become problematic to not only the persons themselves, but also for those around them.

The COMMIT SWELL project [2] aims to develop an unobtrusive monitoring system that will aid a person into improving their health and well-being. Furthermore, health care professionals can use the system to monitor their patients and pose interventions when deemed necessary. As such, the system will aid people both when they use it as a self-help device and as a means of remotely monitoring and supporting patients.

The system envisioned, however, consists of multiple subsystems supplied by a multiplicity of manufacturers. As such, interoperability can not be guaranteed. Furthermore, the maintenance of an application consisting of an increasing number of subsystems is increasingly costly.
Another problem faced in the domain of well-being applications, is the difference between user needs and features provided by the software. The resulting technology push causes user to initially use the system, but fail to incorporate it in their daily life, abandoning usage of it after a period of time.

This paper is structured as follows: Section II discusses our objective, Section III introduces concepts used in our method, which is described in Section IV. Section V lists advantages and disadvantages of our approach. Section VI discusses related work. Conclusions are given in Section VII.

\section{II. ОвJеCTIVE}

The system that is to be created by SWELL will motivate the user to live a healthier life and to provide information regarding the user's body, such that they feel more in control. Lifestyle changes that can be promoted are, for example, exercising more and eating healthier. The user may be rewarded when doing so. Health care professionals will also be able to use the system in order to aid their patients into becoming healthier by monitoring them and imposing interventions.

In order to obtain data from the user, wireless sensors will be utilized, operating in a wireless sensor body area network (BAN). These sensors will relay their data to either a mobile (mobile phone, PDA) or a stationary device (laptop, desktop computer). This device will then visualize the data and using reasoning algorithms give the user advice. Furthermore, the device will transfer and share the obtained data, such that it can be linked to other available data, thus compiling a database which can further be used to enhance the advice.

We propose to use model-driven methods to develop software applications for well-being. Through the use of these techniques, we aim to conquer interoperability issues, improve requirements traceability throughout the development process, and better align user requirements and technology features.

\section{CONCEPTS}

\section{A. Model Transformations}

In model-driven engineering, transformations are defined between two or more models. The transformations denote mappings from elements in the source model to elements in the target model. Through the use of MDE, the speed and quality of the software design process can be increased, as transformations are defined once and can be executed many times. Using MDE 
in the way proposed by the Object Management Group in [3], transformations can be made from high level Computational Independent Models, through Platform Independent Models, into Platform Specific Models. The latter can then be used for code generation. This way of transforming models is referred to as a vertical direction of model transformations

\section{B. Model-Driven Interoperability}

Model-driven interoperability (MDI) uses MDE techniques in order to obtain interoperability between software systems. [4] Instead of vertically, models are said to be transformed horizontally, remaining at the same level of abstraction, but in an other language.

\section{METHOD}

To aid the developers of well-being applications, we will investigate a model-driven way of creating well-being applications. As such, systems consisting of multiple subcomponents will be easier to build. These subcomponents like sensors and devices are not able to communicate initially, but through MDE we can generate interfaces to them, which can in turn be used by the overall application. As a result, the user will not notice the underlying subdivision and the developer will not have to focus on the time consuming activity of interfacing to the subcomponents.

Our MDE approach to developing well-being applications will also incorporate improved requirements alignment. The various subcomponents of a well-being application have various properties and features. As a result, the combination of these components, i.e. the well-being application as a whole, will consist of the sum of these features. Due to the potential number of subcomponents, the relation between the features offered by the application and the requirements of the user is increasingly hard to make. In order to conquer this, our model-driven method will aid requirements alignment.

For the development of our MDE approach, a literary survey shall be conducted to explore the well-being domain and get insights in the current state-of-the-art in MDI. In order to validate our process design, interviews shall be conducted among the stakeholders, workshops shall be organized to retrieve stakeholder requirements and a prototype tool will be created and utilized in a use case to validate its usefulness.

\section{TRADE-OFFS}

The main advantage of using model-driven techniques for developing well-being applications and tracking and aligning requirements, is the high degree of reusability of the developed artifacts. As a result, once created, they may be reused.

The way an MDE process works causes the primary concern, which is the overhead that results from this process. A trade-off has to be made with regard to the prospective size of the project and the time required to develop models and transformations. In other words: for a small application, requiring data from only a select number of sources or sensors, an MDA approach might be regarded as "over engineering", as more time is spent on the models and transformations than on the final product.
In order to make a well thought out decision regarding the use of MDE techniques, estimations regarding the size of the current and future projects will have to be made.

\section{RELATED WORK}

In the U-Care project [5], [6], researchers create a patient centered health care application, aimed at assisting elderly citizens in independent living. Although similarities with SWELL exist from a technological point of view, the target audiences between our project and U-Care is different, as we focus on healthy adults who want to improve their well-being.

Myotel [7] is a project which aims to design a teletreatment system based on myofeedback. Their primary users differ from SWELL: their focus is mainly on users with chronic neck and shoulder complaints, while SWELL's view is broader. Other users exist in the form of health care professionals monitoring these patients.

\section{CONCLUSION AND FUTURE WORK}

As the overall health and well-being of the Dutch population is declining, we propose a pervasive system that will allow users to improve their own lifestyle and situation in order to improve their well-being. Development of these systems may, however, be troublesome due to their complexity.

We propose to use model-driven techniques in order to create systems that aid people in obtaining and attaining a feeling of well-being. Our method will aid developers in creating these applications by improving subsystem interoperability, improve requirements traceability, and better the alignment of user demands and features offered by the system.

\section{ACKNOWLEDGMENT}

We would like to thank Marten van Sinderen for his helpful comments and insights. This work is part of the COMMIT SWELL project.

\section{REFERENCES}

[1] W. Hooftman and et al, "Arbobalans 2010: Kwaliteit van de arbeid, effecten en maatregelen in nederland," TNO, Tech. Rep., 2011.

[2] W. Kraaij, "COMMIT Projectplan, Workpackages, Deliverables, Budget User Centric Reasoning for Well-working (P07)," TNO, Project planning, 2011.

[3] J. Miller and J. Mukerji, "MDA Guide version 1.0.1," Object Management Group, OMG document, 2003. [Online]. Available: http://www.omg.org/cgi-bin/doc?omg/03-06-01

[4] R. Grangel et al, "Transforming GRAI Extended Actigrams into UML Activity Diagrams: a First Step to Model Driven Interoperability," in Enterprise Interoperability II, R. J. Gonçalves, J. P. Müller, K. Mertins, and M. Zelm, Eds. Springer London, 2007, pp. 447-458, 10.1007/978-1-84628-858-6_48. [Online]. Available: http: //dx.doi.org/10.1007/978-1-84628-858-6_48

[5] M. Zarifi Eslami and M. J. van Sinderen, "Flexible home care automation adapting to the personal and evolving needs and situations of the patient," in 3rd International Conference on Pervasive Computing Technologies for Healthcare, 2009. PervasiveHealth 2009, London, UK. Los Alamitos, CA: IEEE, March 2009, pp. 1-2.

[6] J. W. J. R. van't Klooster et al, "Putting the patient at the centre: Making telemedicine services personal again," in Proceedings of the 3rd International Conference on Pervasive Computing Technologies for Healthcare, Pervasive Health 2009, London. Gent: ICST, March 2009, p. 6071.

[7] Myotel: MYOfeedback based TELetreatment service. Roessingh Research and Development. [Online]. Available: http://www.myotel.eu 